\title{
Practical guide to saddle-point construction in lens design
}

\author{
Florian Bociort, Maarten van Turnhout ${ }^{*}$ and Oana Marinescu \\ Optics Research Group, Delft University of Technology \\ Lorentzweg 1, 2628 CJ Delft, The Netherlands
}

\begin{abstract}
Saddle-point construction (SPC) is a new method to insert lenses into an existing design. With SPC, by inserting and extracting lenses new system shapes can be obtained very rapidly, and we believe that, if added to the optical designer's arsenal, this new tool can significantly increase design productivity in certain situations. Despite the fact that the theory behind SPC contains mathematical concepts that are still unfamiliar to many optical designers, the practical implementation of the method is actually very easy and the method can be fully integrated with all other traditional design tools. In this work we will illustrate the use of SPC with examples that are very simple and illustrate the essence of the method. The method can be used essentially in the same way even for very complex systems with a large number of variables, in situations where other methods for obtaining new system shapes do not work so well.
\end{abstract}

Keywords: optical system design, optimization, saddle points

\section{INTRODUCTION}

Saddle-point construction (SPC) is a new method to insert lenses into an existing design. Lens designers frequently insert lenses into their designs and, in the traditional way, one new system shape results after optimization. However, when a lens is inserted with SPC, two distinct system shapes result and for further design one can choose the better one. With SPC, by inserting and then, if necessary, by extracting lenses, new system shapes can be obtained very rapidly. Therefore, we believe that, if added to the optical designer's arsenal, this new tool can significantly increase design productivity in certain situations.

Figure 1 shows a lithographic objective design obtained with SPC. We have also applied SPC successfully in the design of different types of high-NA catadioptric deep UV objectives and of extreme UV mirror systems. As a method for designing lithographic objectives, a patent application for SPC has been submitted. However, the method is generally applicable and for all other types of optical systems, it can be used freely. The authors encourage the interested reader to try this method out.

The SPC method is described in detail in Refs. 1-2. The fact that the theory behind SPC contains mathematical concepts that are still unfamiliar to many optical designers (such as that of a saddle point in a high-dimensional variable space) may unnecessarily deter potential users from trying this new tool out. However, for practical purposes, it is not necessary to have a deep understanding of all of the underlying mathematics. The practical implementation of the method is actually very easy and the method can be fully integrated with all other traditional design tools. (The basic prerequisites will be summarized in Sec. 2.)

In this paper, we will illustrate the use of SPC with five examples (Sec. 3). The first three examples, which form the core of this paper, are very simple and illustrate the essence of the method. The steps given in these examples should be repeated on one's own computer and should then be adapted according to the own needs. The last two examples illustrate the fact that the method can be used essentially in the same way even for very complex systems with a large number of variables, in situations where other methods for obtaining new system shapes do not work so well. The last example also shows that the method is applicable for mirror systems as well.

*m.vanturnhout@tudelft.nl; phone: +31 15278 8109; fax +31 15278 8105;

Current Developments in Lens Design and Optical Engineering VIII, edited by Pantazis Zakos Mouroulis,

Warren J. Smith, R. Barry Johnson, Proc. of SPIE Vol. 6667, 666708, (2007)

0277-786X/07/\$18 - doi: $10.1117 / 12.732477$

Proc. of SPIE Vol. 6667 666708-1 


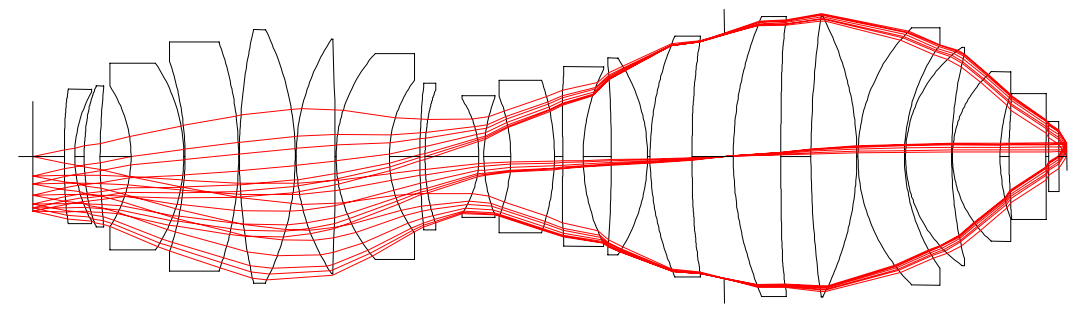

Fig. 1. At a numerical aperture of 0.85 and the wavelength of $193 \mathrm{~nm}$, this system with 107 variables has a wavefront aberration of $2.37 \mathrm{~m} \lambda$ (Strehl ratio of 0.9998 ) and a distortion below $1 \mathrm{~nm}$. For details see Ref. 3.

\section{SPC IN A NUTSHELL}

For simplicity, we discuss here the case when the element to be inserted is a lens with spherical surfaces. The method also works with mirrors and aspherical surfaces, see our papers ${ }^{2,3}$ and the last example.

We start with an optimized system with $N$ spherical surfaces. The curvatures of the surfaces are used as variables. Any optical merit function can be used, e.g. a merit function based on transverse aberrations (root-mean-square spot size), wavefront aberration, etc.

In the existing system, which is a local minimum with $N$ surfaces, we insert a meniscus lens with zero thickness and equal curvatures. Such a meniscus disappears physically (!) and does not affect the path of any ray or the merit function of the system. We call this meniscus a "null-element". One may wonder, if a lens does nothing, why is it needed? Actually, the "null-element" comes into play when the new system is slightly changed: the "null-element" comes with two new variables - the two surface curvatures, and for some specific values for these curvatures, it transforms the local minimum into a "saddle point" in the variable space with increased dimensionality. As shown below, this enables then the merit function to decrease. The full details can be found in Ref. 1.

An intuitive illustration of SPC is given in Figure 2. The starting system is a minimum in all variables. For simplicity, only one variable is shown in red (dark gray) in the upper left part of Figure 2. When the "null-element" is added, a new "downward" direction, shown in green (the lighter gray curve passing through the saddle point), and an "upward" direction (not shown) appear in the new variable space (with a dimension increased by 2). Along the downward direction the new system is a maximum. In fact, the new system is a saddle point, which we call a "null-element" saddle point (NESP). Despite of the fact that typically we have much more than two variables, the NESP resembles very much a twodimensional horse saddle. If we choose two points close to the NESP, but situated on opposite sides of the saddle, and then optimize them, the optimizations "roll down" from the NESP and arrive at two distinct local minima. (The optimization variables are those of the starting local minimum plus the two curvatures of the "null-element".)

In the general case, when the insertion position and the glass of the "null-element" are arbitrary, the curvature of the "null-element" meniscus can be computed numerically ${ }^{4}$. A very powerful version of the method is the one in the special case when the "null-element" is inserted in contact with (i.e. at zero axial distance from) an existing surface (called reference surface) in the original local minimum. Also, the glass of the "null-element" should be the same as the glass at the reference surface. In this special case, the two curvatures of the "null-element" are equal to the curvature of the reference surface ${ }^{1}$ (see Figure 3). Thus, at the insertion position we have three surfaces with equal curvatures and with two zero distances between them. 


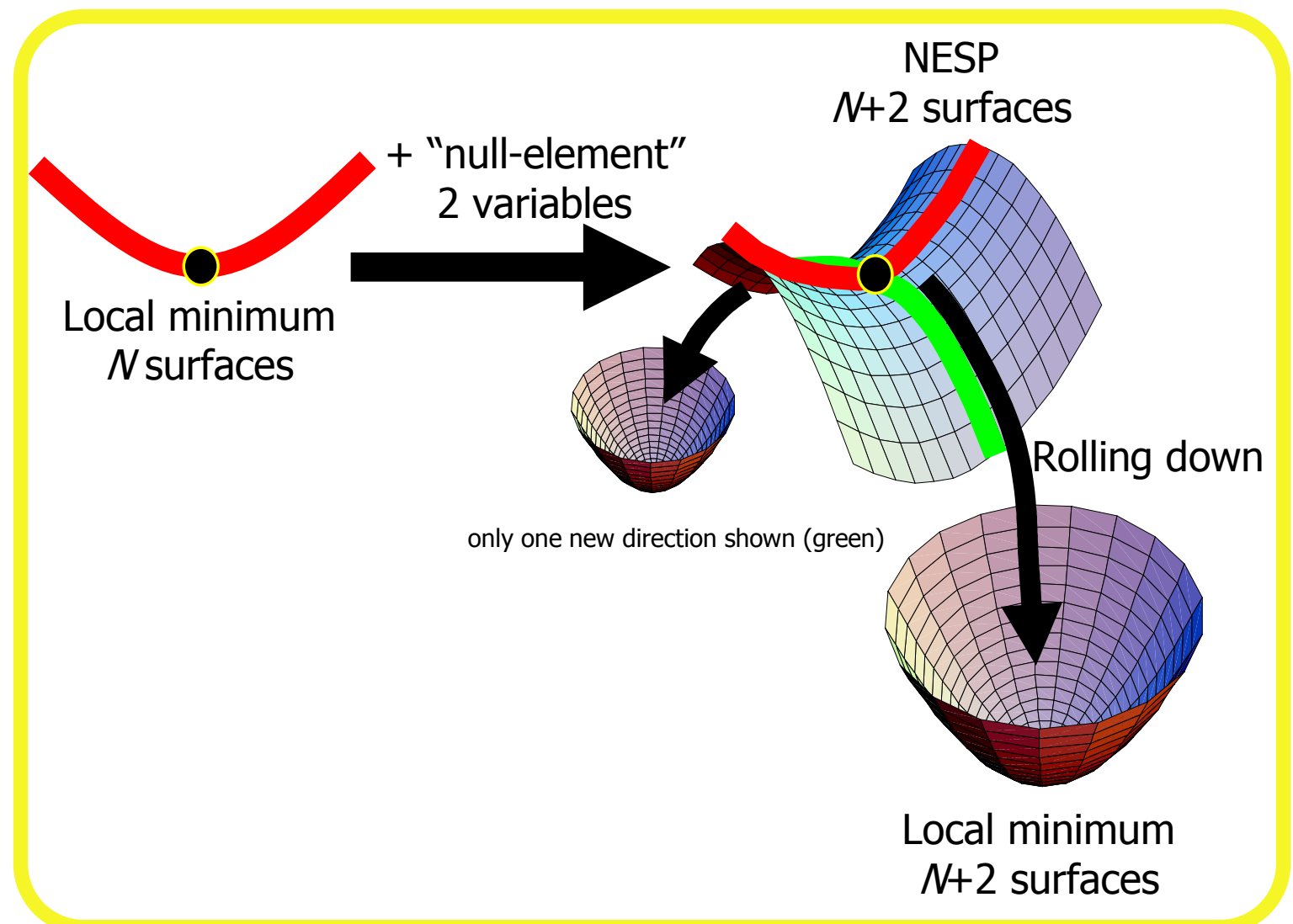

Fig. 2. Obtaining two local minima with $N+2$ surfaces from a local minimum with $N$ surfaces via a NESP.

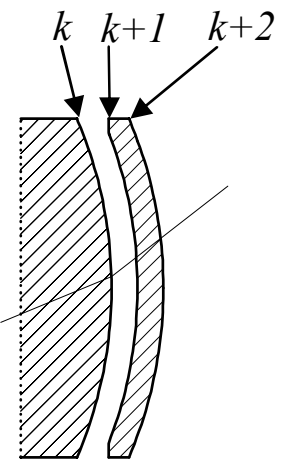

Fig. 3. A NESP with three consecutive surfaces having equal curvatures, $\mathrm{c}_{k}=\mathrm{c}_{k+1}=\mathrm{c}_{k+2}$. In this drawing, the meniscus with surface curvatures $\mathrm{c}_{k+1}$ and $\mathrm{c}_{k+2}$ is inserted after the reference surface $k$. For clarity, the two zero distances between these surfaces are shown as small nonzero distances. If the meniscus is inserted before the reference surface, then the reference surface has in the new system the index $k+2$.

After a NESP is constructed as described above, we take two points on opposite sides of the saddle, by slightly changing two consecutive surfaces, which are in contact in the NESP:

$$
\mathrm{c}_{k+1}=\mathrm{c}_{k+2}=\mathrm{c}_{r e f} \pm \varepsilon, \quad \mathrm{c}_{k}=\mathrm{c}_{r e f}
$$

or alternatively 


$$
\mathrm{c}_{k}=\mathrm{c}_{k+1}=\mathrm{c}_{r e f} \pm \varepsilon, \quad \mathrm{c}_{k+2}=\mathrm{c}_{r e f}
$$

where $c_{r e f}$ is the curvature of the reference surface in the starting local minimum, and $\varepsilon$ indicates a small change in the surface curvatures. (The procedure remains valid if radii are used instead of curvatures.)

By optimizing the two points obtained either with (1) or with (2), we obtain two different local minima. If $\varepsilon$ is chosen in the correct range, the same pair of local minima is obtained from (1) and (2). For this reason, using only one pair of points, either (1) or (2), is sufficient.

Once the two minima on both sides of the NESP have been obtained, the distances between surfaces and the glass of the lens resulting from the "null-element" can be changed as desired.

\section{EXAMPLES}

The special case of the SPC method discussed above is illustrated below with five examples. The specific part of the SPC (i.e. constructing the NESP and obtaining the four points close to the saddle) can be executed in a very short time in all examples. In these examples we optimize only one pair of points (1) or (2). After optimizing the two points, in the resulting local minima the zero distances between surfaces inherited from the "null-element" are increased gradually to the desired values. The steps should be small enough to avoid jumps to other local minima. This can take a longer time, especially for complex optical systems such as those in the last two examples, where local optimization is timeconsuming.

The reader is encouraged to reproduce the first and third example entirely and at least parts of the second one, in order to see how the method works. For the first three examples the necessary steps are given in detail, so that they can be reproduced with any optical design software. We have used $C O D E V$, but have tested the first three examples in $Z E M A X$ as well, obtaining the same results. For $C O D E V$, lens files for all steps and a macro that creates the NESP are available via our website ${ }^{5}$. Certain steps given in the examples can then be reproduced automatically. If desired, for obtaining the starting configurations, users of other optical design software can import or adapt the corresponding $C O D E V$ files, which are in ASCII format. Automatic conversion to the format of another program is very useful and does most of the work, but sometimes some information (e.g. wavelengths, solves) is lost. Therefore, for the present purpose it is important to compare the result of automatic conversion with the information given in the tables below, and to make changes wherever necessary.

\subsection{Example 1. Obtaining a doublet from a singlet}

This very simple example serves two purposes: it illustrates the special case of the SPC method, which can be used in essentially the same way in all cases, and also shows an important advantage of SPC. When using SPC to insert a lens so that a NESP is created, two systems result after optimization. Inserting or splitting a lens in the traditional way results in a single system, which is not necessarily the best one. In this example, the better solution obtained from a singlet with SPC is missed when splitting the same singlet in a traditional way.

In five steps, we obtain two doublet local minima by starting from a singlet minimum. The curvatures are used as variables. In the first three examples, the merit function is based on transverse aberrations (root-mean-square spot size) with respect to the chief ray. In this example, it also includes as constraint a constant effective focal length. In the merit function, all wavelengths and fields have a weight factor of unity. The distance between the last surface and the image has a paraxial image distance solve.

The singlet that we have used as starting system has the following specifications:

Table 1. Starting system for Example 1.

\begin{tabular}{|c||c|c|c|c|}
\hline \multicolumn{1}{|c||}{ Surface \# } & Radius $(\mathrm{mm})$ & Thickness (mm) & Glass type \\
\hline \hline 1 (STOP) & 59.33336 & $\mathrm{~V}$ & 4.000000 & BK7_SCHOTT \\
\hline 2 & -391.44174 & $\mathrm{~V}$ & 97.703035 & AIR \\
\hline
\end{tabular}


The object is placed at infinity. All thicknesses and glass types are constant. The variable radii of curvature are indicated with "V".

Table 2. Specifications for the systems in Example 1.

\begin{tabular}{|l|l|}
\hline Entrance pupil diameter $(\mathrm{mm})$ & 20.00000 \\
\hline Effective focal length (mm) & 100.0000 \\
\hline Paraxial image height (mm) & 8.7489 \\
\hline $\begin{array}{l}\text { Wavelengths (nm) } \\
(*)=\text { reference }\end{array}$ & $656.30,587.60(*)$, and 486.10 \\
\hline Fields (deg) & $0.00000,3.00000$, and 5.00000 \\
\hline
\end{tabular}

(We have worked in $C O D E V$ with curvatures, but we list in our examples the radii, because it seems that in some other programs it is easier to work in this way.)

In the first three examples, the control of edge thickness violation was disabled when optimizing the two starting points situated on both sides of the saddle. With two zero thicknesses, an edge thickness violation can easily appear, but may disappear when the zero thicknesses are increased in the resulting local minima.

The procedure is as follows:

Step 1: $\quad$ Start with the singlet given in Table 1 and reoptimize.

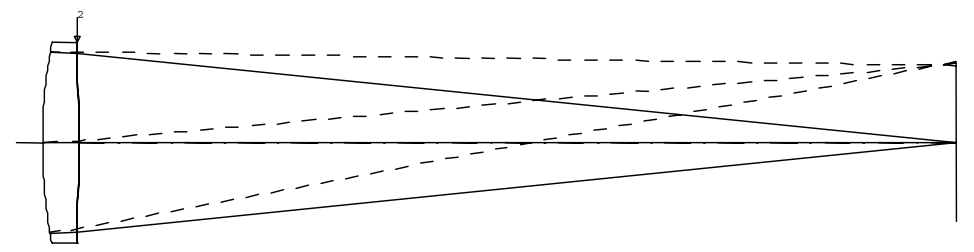

Fig. 4. Starting system for Example 1.

Step 2: $\quad$ Insert a "null-element" (a meniscus with zero thickness) in contact with the second surface (with curvature $c_{2}$ ) of the singlet (the arrow in Fig. 4). The glass of the new lens must be the same as that of the first one. Next, make the two curvatures $\left(c_{3}\right.$ and $\left.c_{4}\right)$ of the "null-element" equal to $c_{2}$. The obtained system is a doublet NESP. Finally, make the new curvatures variable. (Note that, because of the zero distances and the property $c_{2}=c_{3}=c_{4}$, the lens drawing for the NESP is indistinguishable from the one for the original local minimum shown in Fig. 4.)

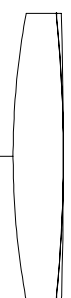

a ) (1)

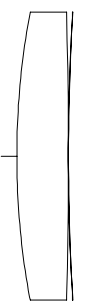

(2)

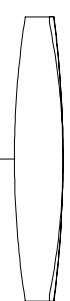

b) (1)

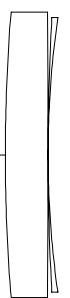

(2)

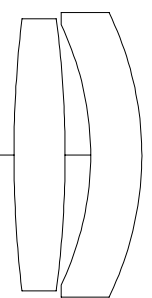

c) (1)

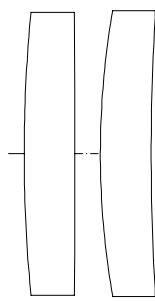

(2)

Fig. 5. Steps for SPC in Example 1. a) Step 3, b) step 4, c) step5.

Step 3: While keeping the first two curvatures of the doublet (i.e. the old variables of the singlet) unchanged, change the two curvatures of the thin lens. Construct one doublet with the property $c_{3}=c_{4}=c_{2}-\varepsilon$ (system 1, Fig. 5a), and one doublet with the property $c_{3}=c_{4}=c_{2}+\varepsilon$ (system 2, Fig. 5a), where $\varepsilon$ indicates a small curvature change. To avoid that the gradient of the merit function becomes too small for optimization, $\varepsilon$ should not be chosen too small. In this example, $\varepsilon=0.00003 \mathrm{~mm}^{-1}$ (in the figure, the curvature change is shown exaggerated). In this way, two systems situated on opposite sides of the saddle are obtained which can be further optimized to generate two new solutions. 
Step 4: $\quad$ Optimize the two systems that have been obtained after step 3. The result is shown in Fig. 5 b.

Step 5: Gradually increase the thickness of the lens resulting from the "null-element" and the axial distance between it and the previous lens, until the desired values are obtained. In this example, the thickness of the "null-element" has been increased in four steps (e.g. thickness $=1,2,3$, and then $4 \mathrm{~mm}$ ) and the air distance separating the two lenses of the system in two steps (e.g. distance =1, and then $2 \mathrm{~mm}$ ). After each increment, the system should be reoptimized (Fig. 5c).

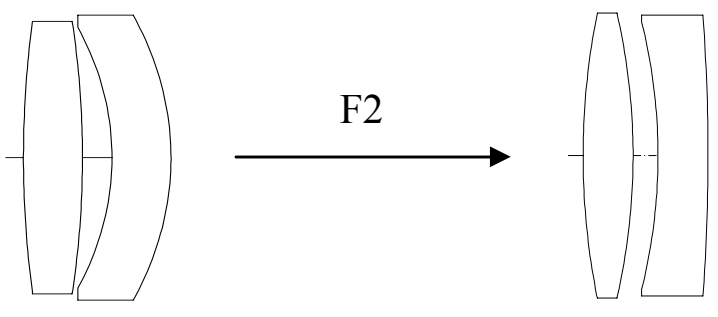

Fig. 6. Obtaining a Fraunhofer-type design.

Since the two glasses of the doublets resulting from step 5 are the same, these systems are not corrected for axial color. However, by glass change we can improve the first of the two systems. For example, if we change the material of the second lens from BK7 to F2, system 1 will become after reoptimization a well-known air-spaced Fraunhofer-type design.

When the starting singlet is split in a traditional way as shown below and then reoptimized, the resulting system will be system 2 in step 5. However, this system has a poorer imaging performance than system 1 in step 5, which can lead to the Fraunhofer-type configuration. This is illustrated below:

Step 1': $\quad$ Start with the optimized singlet used for the SPC (Fig. 4 ).

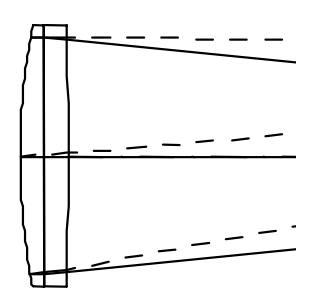

(a)

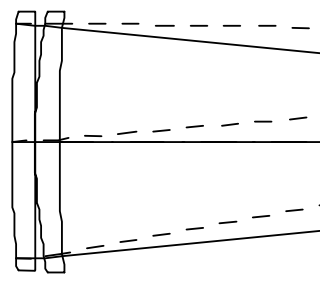

(b)

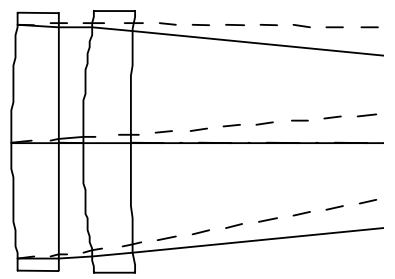

(c)

Fig. 7. Traditional power splitting. a) Step 2', b) step 3', c) step 4'.

Step 2': $\quad$ Split the singlet into two lenses with the same thickness (i.e. insert a plate of air with zero thickness and flat surfaces in the middle of the singlet, see Fig. 7a).

Step 3': $\quad$ Optimize the system with the curvatures as variables. (The merit function remains the same as above.) The result is shown in Fig. $7 b$.

Step 4': $\quad$ Increase the thickness of the two lenses and the axial distance between them to the same values as that in the two systems obtained with SPC (see step 5 above). (In our example, the lens thickness is $4 \mathrm{~mm}$ and the air distance is $2 \mathrm{~mm}$, see Fig. 7c.)

The final system that results from splitting the singlet is one of the two systems which are obtained with SPC (system 2 in Fig. 5c). It has a merit function that is worse than that of the other system obtained with SPC (system 1 in Fig. 5c).

\subsection{Example 2. Obtaining a quintet from a quartet}

In this example, we observe a property ("convergence") that occurs frequently with SPC: the same final design can be obtained in several different ways. (See e.g. Figure 3 of Ref. 2 where the same DUV lithographic design is obtained in six different ways from the same precursor system with less lenses, or Figure 4 of Ref. 3, where the same eight-mirror 
EUV lithographic design -m8- was obtained with SPC from two very different six-mirror precursors -m6S2A and m6S3A.) This is important because if for any reason a design route, that should be successful, accidentally misses the goal (e.g. for sufficiently complex systems, sometimes even the local optimization details influence the outcome of local optimization), in many cases the same goal can be achieved via another design route of the same kind.

Starting with a monochromatic quartet, in this example we will construct quintet NESP's in three different ways with the procedure described in Section 2. We will see that out of the six resulting quintet minima, three of them (one resulting from each NESP) are identical.

The quartet used as starting system has the following specifications:

Table 3. Starting system for Example 2.

\begin{tabular}{|c||c|c|c|c|}
\hline \multicolumn{1}{|c||}{ Surface \# } & Radius $(\mathrm{mm})$ & Thickness $(\mathrm{mm})$ & Glass type \\
\hline \hline 1 & 50.47190 & $\mathrm{~V}$ & 12.000000 & SK1_SCHOTT \\
\hline 2 & 132.70940 & $\mathrm{~V}$ & 0.000000 & AIR \\
\hline 3 & 32.40194 & $\mathrm{~V}$ & 12.000000 & SK1_SCHOTT \\
\hline 4 & 22.07294 & $\mathrm{~V}$ & 15.107864 & AIR \\
\hline $5($ STOP $)$ & INFINITY & & 12.921199 & AIR \\
\hline 6 & -25.50939 & $\mathrm{~V}$ & 12.000000 & SK1_SCHOTT \\
\hline 7 & -30.38610 & $\mathrm{~V}$ & 0.000000 & AIR \\
\hline 8 & 149.05514 & $\mathrm{~V}$ & 12.000000 & SK1_SCHOTT \\
\hline 9 & -111.92678 & $\mathrm{~S}$ & 60.005333 & AIR \\
\hline
\end{tabular}

The object is at infinity, and all thicknesses and glass types are constant. The variable radii of curvature are indicated with "V". The merit function is the same as in Example 1, excepting that to keep the effective focal length constant, instead of a constraint within the merit function, the last surface has a solve for the marginal ray exit angle (equal to 0.25 , indicated with "S"). The distance between the last surface and the image has a paraxial image distance solve.

Table 4. Specifications for the systems in Example 2.

\begin{tabular}{|l|l|}
\hline Entrance pupil diameter $(\mathrm{mm})$ & 50.00000 \\
\hline Effective focal length $(\mathrm{mm})$ & 100.0004 \\
\hline Paraxial image height (mm) & 24.9329 \\
\hline Wavelength (nm) & 656.30 \\
\hline Fields (deg) & $0.00000,10.00000$, and 14.00000 \\
\hline
\end{tabular}

First, we construct a quintet NESP by inserting a "null-element" at the second surface in this quartet.

Step 1: $\quad$ Start with the quartet given in Tab. 3 and reoptimize.

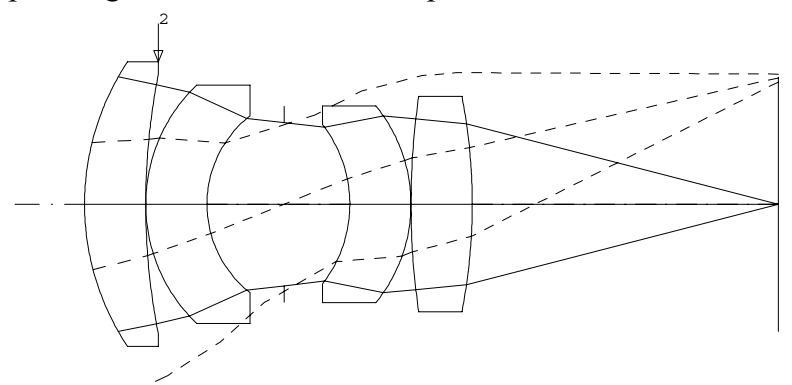

Fig. 8. Starting system for Example 2.

Step 2: Insert a "null-element" (with variable curvatures) in contact with the second surface of the quartet (arrow 2 in the Fig. 8). The surface curvatures of the "null-element" are $c_{3}$ and $c_{4}$, and that of the surface it is in contact with is $c_{2}$. Make $c_{3}$ and $c_{4}$ equal to $c_{2}$ and the glass of the "null-element" the same as that of the first lens. The obtained system with $c_{2}=c_{3}=c_{4}$ is a (quintet) NESP. 


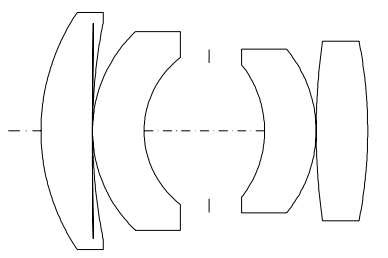

(a1)

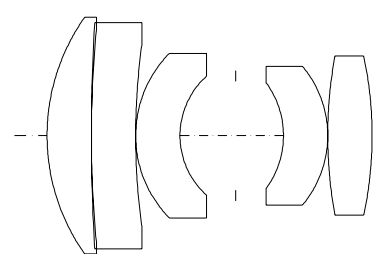

$(\mathrm{c} 1)$

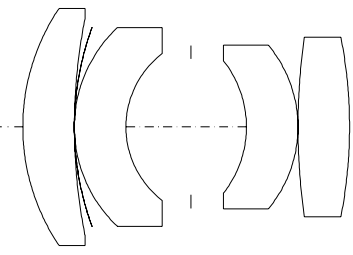

(a2)

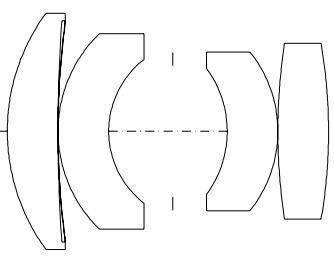

(b1)

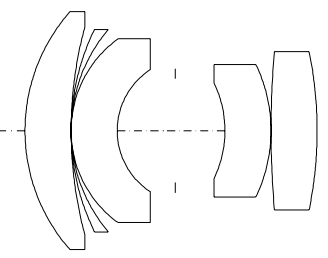

(b2)

Fig. 9. Steps for SPC in Example 2. a) Step 3, b) step 4, c) step 5, d) edge separation violation removed in system (c1).

Step 3: While keeping the "old" variables of the quartet unchanged, change the two curvatures of the "nullelement" as follows: $c_{3}=c_{4}=c_{2}-\varepsilon$ and $c_{3}=c_{4}=c_{2}+\varepsilon$, where $\varepsilon$ indicates a small curvature change. In this example $\varepsilon=0.00003 \mathrm{~mm}^{-1}$ (in Figs. 9a1 and 9a2, the curvature change is shown exaggerated). In this way, two systems on opposite sides of the saddle are obtained.

Step 4: $\quad$ Optimize the two systems that have been obtained after step 3. The result is shown in Figs. 9b1 and $9 \mathrm{~b} 2$.

Step 5: In the resulting systems, gradually increase the thickness of the "null-element" (i.e. increase the thickness in small steps and optimize the system after each step) until the desired values are obtained. For this example, the thickness of the "null-element" has been increased in eight steps (e.g. thickness = $1,2,3,4,6,8,10$, and then $12 \mathrm{~mm}$ ). After each increment, the system should be reoptimized (a few cycles are usually sufficient in the intermediate stages). To remove the edge separation violation in system 9c1, we slightly increase the axial distance between the first two lenses. (Here this distance was set to $3 \mathrm{~mm}$.)

As an exercise, we invite the reader to repeat the steps 1-5 described above at the third surface of the quartet. For verification, the answer is given in our web document ${ }^{5}$.

When comparing the four systems obtained from these two NESP's, we observe that two of the systems (the system in Fig. 9c2 and system 1 after step 5 in Appendix B of Ref. 5) are identical.

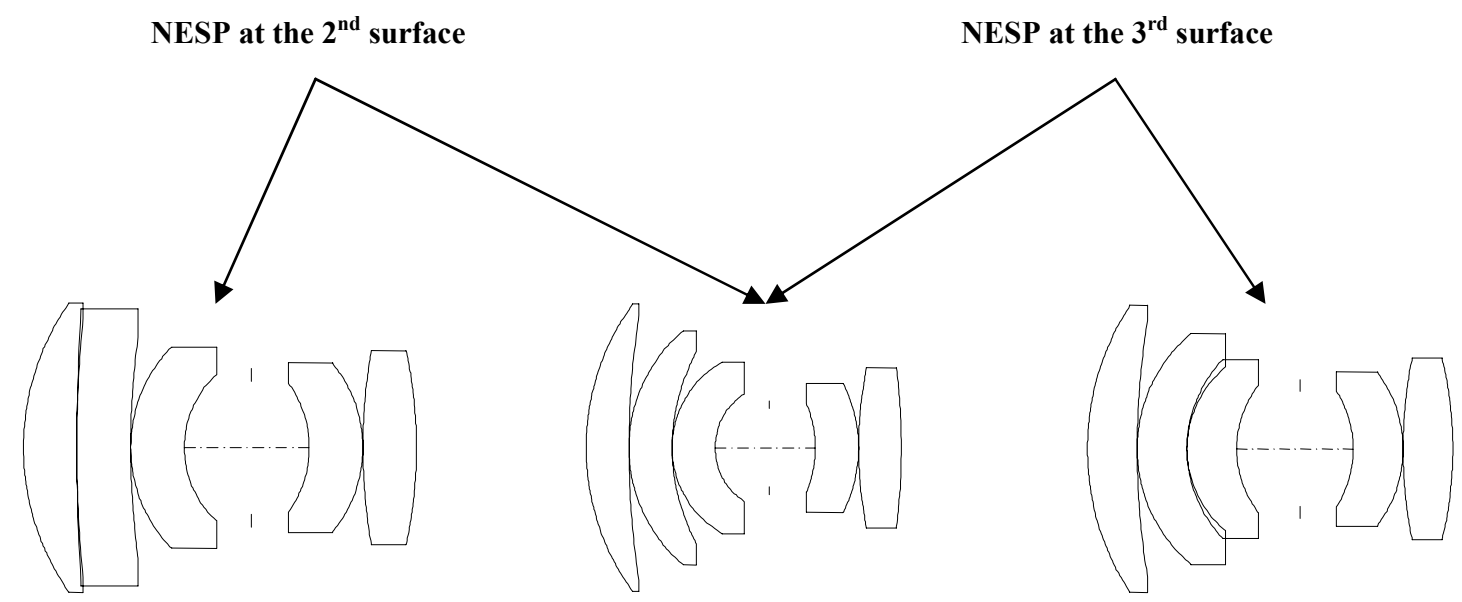

Fig. 10. The middle system in this drawing can be obtained from both NESP's. 
The middle system in Figure 10 (we call it a "hub") can be obtained from other NESP's as well. For instance, by inserting a "null-element" lens at the first surface of the quartet, concentric with that surface and with the same glass, we obtain a third NESP. For obtaining the "hub" we choose the side of the NESP where the meniscus lens has a slightly weaker curvature than that of surface 1, as in Figure 11a. After optimization we obtain the quintet shown in Figure 11b.

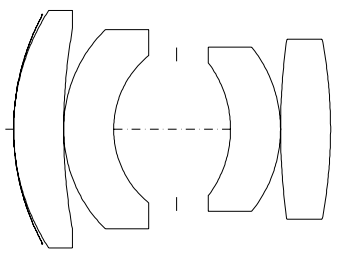

(a)

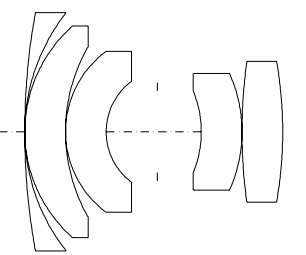

(b)

Fig. 11. a) Perturbed NESP at first surface. b) Perturbed NESP optimized.

In the original quartet all lenses have the same thickness. After increasing the thickness of the inserted lens in Fig. 11b to the same value as for the other lenses and reoptimizing, we obtain the "hub" (Fig. 9c2) again.

\subsection{Example 3. Obtaining a better system shape by inserting and extracting lenses}

Frequently, experienced designers observe that the shape of a design is probably not the best possible one. Here we show how we can move from one local minimum to a better one by first inserting, and then by extracting a lens. First, we use the SPC method at the second surface of the starting system to construct a NESP. After optimizing it on one of its two sides, we obtain a local minimum. Then, by removing the first lens and reoptimizing, we obtain a local minimum with the same number of lenses as the starting one, but with a much lower merit function value. The final shape resembles the well-known Double Gauss design.

The system parameters of the starting system are:

Table 5. Starting system for Example 3.

\begin{tabular}{|c||c|c|c|c|}
\hline Surface \# & Radius $(\mathrm{mm})$ & Thickness (mm) & Glass type \\
\hline \hline 1 & -163.22088 & $\mathrm{~V}$ & 8.746658 & BSM24_OHARA \\
\hline 2 & -186.19903 & $\mathrm{~V}$ & 0.298182 & AIR \\
\hline 3 & 29.70917 & $\mathrm{~V}$ & 12.424230 & SK1_SCHOTT \\
\hline 4 & INFINITY & & 3.776966 & F15_SCHOTT \\
\hline 5 & 39.76096 & $\mathrm{~V}$ & 15.107864 & AIR \\
\hline $6($ STOP $)$ & INFINITY & & 12.921199 & AIR \\
\hline 7 & -16.65265 & $\mathrm{~V}$ & 3.776966 & F15_SCHOTT \\
\hline 8 & INFINITY & & 10.833928 & SK16_SCHOTT \\
\hline 9 & -29.22267 & $\mathrm{~V}$ & 0.298182 & AIR \\
\hline 10 & 283.14792 & $\mathrm{~V}$ & 6.858175 & SK16_SCHOTT \\
\hline 11 & -64.40364 & $\mathrm{~S}$ & 72.362756 & AIR \\
\hline
\end{tabular}

The object is placed at infinity, and all thicknesses and glass types are constant. The variable radii of curvature are indicated with "V". The merit function is the same as in Example 2, and the last surface has a solve for the marginal ray exit angle (equal to -0.15 , indicated with " $\mathrm{S}$ "). The distance between the last surface and the image has a paraxial image distance solve.

Table 6. Specifications for the systems in Example 3.

\begin{tabular}{|l|l|}
\hline Entrance pupil diameter $(\mathrm{mm})$ & 30.00000 \\
\hline Effective focal length (mm) & 100.0000 \\
\hline Paraxial image height (mm) & 24.9328 \\
\hline $\begin{array}{l}\text { Wavelength (nm) } \\
(*)=\text { reference }\end{array}$ & $656.30,587.60(*)$, and 486.10 \\
\hline Fields (deg) & $0.00000,10.00000$, and 14.00000 \\
\hline
\end{tabular}


Step 1: $\quad$ Start with the quartet given in Tab. 5 and reoptimize.

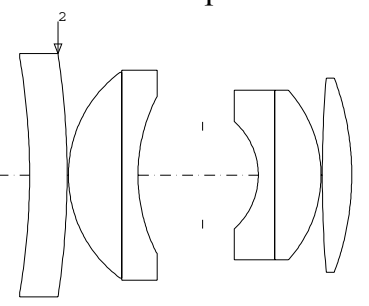

Fig. 12. Starting system for Example 3.

Step 2: Insert a "null-element" (with variable curvatures) in contact with the second surface of the quartet, indicated with arrow 2 in the Fig. 12. Use for the "null-element" the glass of the first lens.

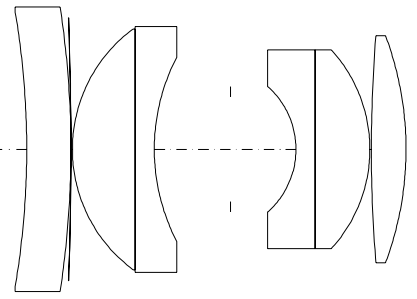

(a)

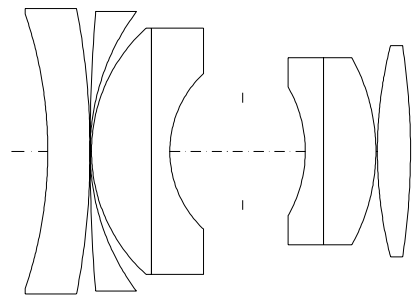

(b)

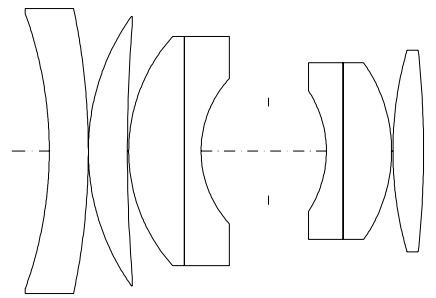

(c)

Fig. 13. Steps for SPC in Example 3. a) Step 3, b) step 4, c) step5.

Step 3: Change the two curvatures of the "null-element" as follows: $c_{3}=c_{4}=c_{2}+\varepsilon$, where $\varepsilon=0.00003 \mathrm{~mm}^{-1}$ (i.e. reduce the negative curvature; when working with radii, make the two radii a negative number with larger absolute value). For clarity, the curvature change is shown exaggerated in Fig. 13a.

Step 4: $\quad$ Optimize the system that has been obtained after step 3 (Fig. 13b).

Step 5: Increase the thickness of lens resulting from the "null-element" to the same value as that of the first lens (here, this can be done in one step), and reoptimize. The result is shown in Fig. 13c.

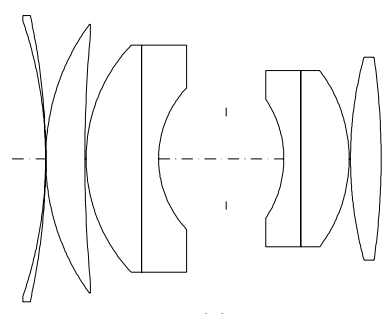

(a)

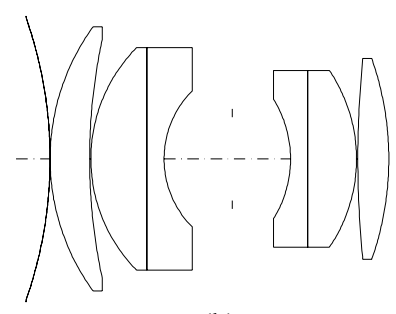

(b)

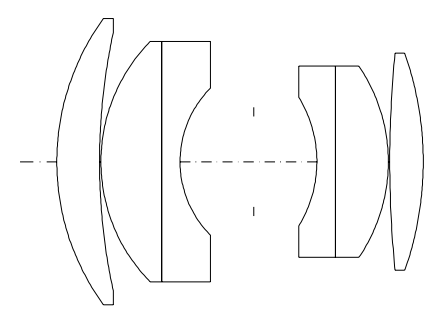

(c)

Fig. 14. Extracting a lens. a) Step 6, b) step 7, c) step8.

Step 6: $\quad$ Decrease the thickness of the first lens to zero, and reoptimize (Fig. 14a).

Step 7: $\quad$ Make the curvature of the second surface equal to the one of the first surface, and reoptimize the system with the first two curvatures fixed (Fig. 14b).

Step 8: $\quad$ Delete the first lens, and reoptimize the system. The resulting system (Fig. 14c) has a merit function that is much lower than that of the starting one and the shape resembles the well-known Double Gauss design.

We have applied similar techniques successfully in the design of state-of-the-art lithographic designs. Sometimes, after SPC insertion some lens in the resulting system seems to have no role any more. Such a lens is then a good candidate for removal, even when it is situated farther away than in the above case from the position of SPC insertion. 


\subsection{Example 4. Deep UV lithography}

The special case of the SPC method can be used in essentially the same way in the design of complex optical systems, such as those used in lithography. In this example, the method has been used in the second bulge of a lithographic objective $^{2}$.

A NESP has been constructed at surface 37 using the SPC method. From the NESP, by means of local optimization performed on each side of the saddle, two new systems have been generated. Figure 15 shows the solutions after the thickness of the lens resulting from the "null-element" has been increased. Most differences between the two new systems are observed close to the position where SPC has been used. A detailed study shows that one of these two systems (the lower left one in Fig. 15) can be obtained in at least six different ways from the same starting system (upper drawing) ${ }^{2}$. Since it can be obtained from many other NESP's as well, as in the case of the quintet in Fig. 9c2, we call this system with 44 surfaces a "hub" (i.e. the result of several converging design paths). In fact, the starting system which is a high-quality patented design ${ }^{6,7}$, turns out to be also a hub .

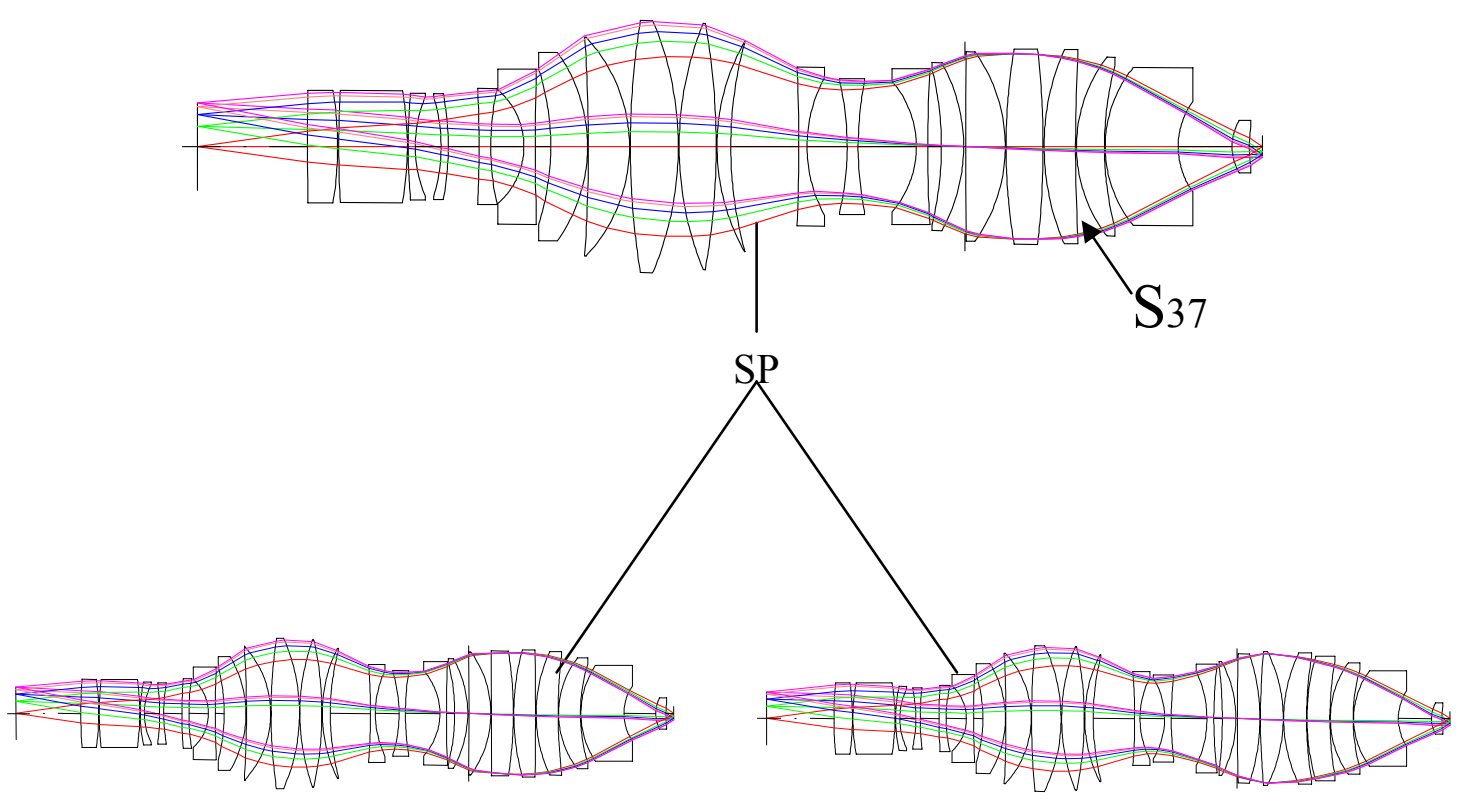

Fig. 15. Generating two configurations for a lithographic objective with 44 surfaces via SPC from a system with 42 surfaces. The numerical aperture is 0.56 , the image height is $11 \mathrm{~mm}$, the magnification is -0.25 and the wavelength is $248 \mathrm{~nm}$.

\subsection{Example 5. Mirror systems for extreme UV lithography}

This example shows that SPC can be used for mirror systems as well and that high-quality designs can be obtained by combining SPC with traditional design techniques.

In the ring-field six-mirror system for extreme UV lithography shown in the upper part of Figure 16, a pair of spherical mirrors has been inserted after the second (spherical) surface. From the two points close to, but on opposite sides of the constructed NESP, two eight-mirror solutions have been obtained by means of local optimization, each having three consecutive mirrors in contact. After increasing the zero axial distances between the mirrors, the solutions have been locally optimized with all variables (curvatures, aspheric coefficients and distances) and practical constraints. In this way, the two systems shown in the lower part of Figure 16 have been obtained. 
The process of generating the two local minima and increasing the axial distances between the three consecutive mirrors is time consuming, and depending on the precise succession of steps and even on local optimization and merit function details, the results might be slightly different from the ones given here. In our approach, in order to succeed increasing the axial distances between the mirrors, the first two surfaces in the system had to be temporarily frozen. They were released when the axial distances reached the desired value and then the systems were reoptimized.

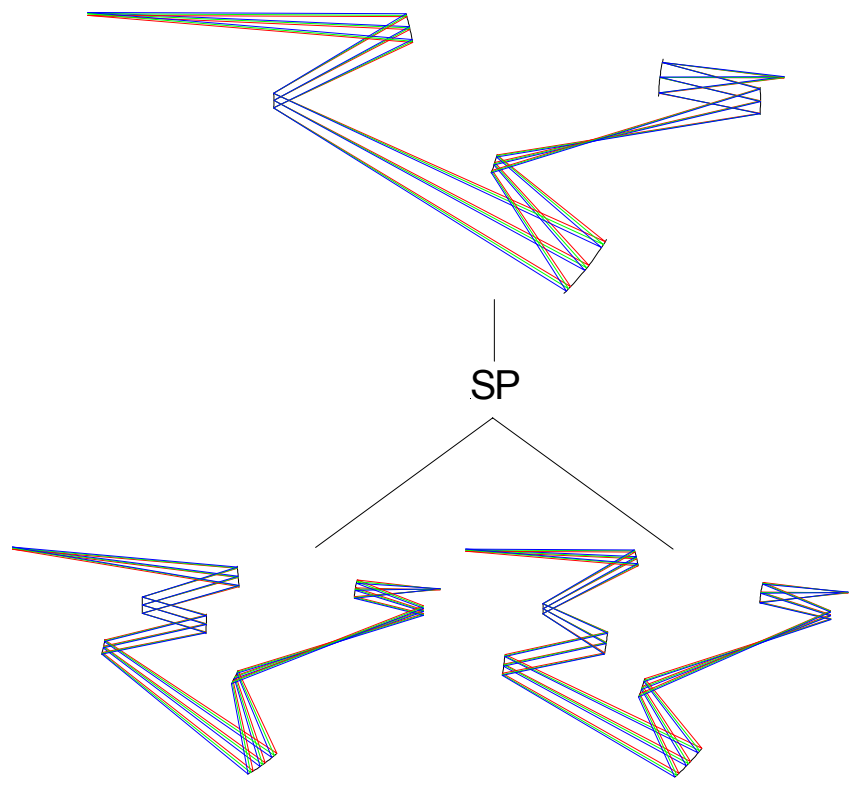

Fig. 16. A NESP has been constructed from the six-mirror system by inserting a pair of mirrors at the second surface. Solutions with eight surfaces have been obtained.

In all cases of extreme UV projection systems we have studied, when increasing the axial distances between the three mirrors in contact in the systems obtained from the NESP, it was important to freeze the two surfaces situated in front of the inserted "null-element". Otherwise, the local optimization process became unstable.

Using traditional design techniques, one of the two solutions generated from the NESP has been further optimized and the numerical aperture has been increased from 0.16 to 0.4 (see Figure 17). All surfaces are aspheric. The resulting system satisfies practical requirements: telecentricity on the wafer side, distortion smaller than $1 \mathrm{~nm}$ per field and Strehl ratio larger than 0.996 with a wavefront aberration of $10 \mathrm{~m} \lambda$ at a wavelength of $13 \mathrm{~nm}$.

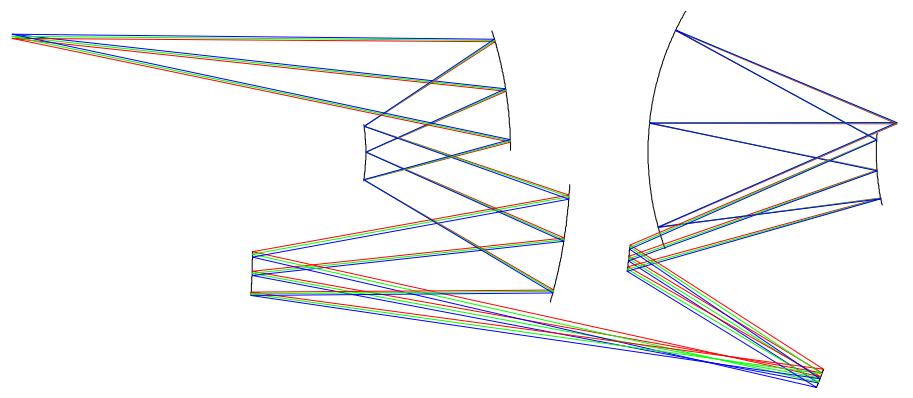

Fig. 17. 0.4 NA projection system for extreme UV lithography.

The SPC method has been used at aspherical surfaces in extreme UV projection systems as well. ${ }^{3}$ 


\section{CONCLUSIONS}

We have seen how we can transform a local minimum into a saddle point by adding a null element. Two starting points for further optimization on opposite sides of the saddle can then be found by using either Eq. (1) or Eq. (2). The original local minimum should be properly optimized so that the residual gradient of the merit function is sufficiently close to zero. A saddle point also has zero gradient, and therefore for typical small values of $\varepsilon$ the two starting points on both sides of the saddle have a relatively small value of the gradient. In order to obtain the desired outcome of the subsequent local optimization, the residual gradient of the original local minimum must be significantly smaller than the $\varepsilon$-dependent gradient at the two starting points.

There are other ways to find such a pair of starting points, but Eqs. (1) and (2) have the advantage that in both systems the newly inserted component is still a null element which does not affect the ray paths. Therefore, if the original local minimum already satisfies the required optimization constraints, the starting points obtained with Eq. (1) or Eq. (2) will satisfy those constraints automatically. However, there is a caveat about this way of choosing starting points on both sides of the saddle. The saddle points are special points on the border between the basins of attraction that correspond to the two adjacent local minima. (The basin of attraction is the set of starting points that after local optimization lead to the same minimum.) Ideally, the two starting points should be points situated deeper within the two basins. However, depending on implementation details of the local optimization algorithm, we have found examples (to be published) where the two starting points are also close to the border. In such cases the outcome of local optimization started at those points can become less predictable. However, when this problem occurs, typically only one pair of points seems to be affected, either the one obtained with Eq. (1) or the one with Eq. (2). The other pair is still well-behaved and is adequate for SPC.

We have successfully used the SPC method for changing system shapes in the design of lithographic objectives. Considering the total amount of effort required for designing a deep UV or extreme UV objective, we have observed in all cases that changing the system shape with our method could be done in a time that was short in comparison with the time we have spent for further refining the new configuration with traditional techniques, including local optimization, to meet practical requirements. Inserting and extracting lenses as shown in this paper has revealed unexpected potential of further improvement even for designs that already had a high quality. However, the method should be applicable in the design of other optical systems as well.

We acknowledge the use of an educational license of CODE V.

\section{REFERENCES}

1. F. Bociort and M. van Turnhout, Generating saddle points in the merit function landscape of optical systems, Proc. SPIE 5962, 0S1-8 (2005)

2. O. Marinescu and F. Bociort, Saddle points in the merit function landscape of lithographic objectives, Proc. SPIE 5962, 0T1-8 (2005)

3. O. Marinescu and F. Bociort, Designing lithographic objectives by constructing saddle points, Proc. SPIE 6342, 6342L (2006)

4. F. Bociort and M. van Turnhout, Looking for order in the optical design landscape, Proc. SPIE 6288, 628806, (2006)

5. http://wwwoptica.tn.tudelft.nl/users/bociort/SPC_guide.zip

6. T. Sasaya, K. Ushida, Y. Suenaga, and R. I. Mercado, Projection optical system and projection exposure apparatus, U.S. Patent 5,805,344 (1998).

7. J. B. Caldwell, All-fused silica 248-nm lithographic projection lens, Optics and Photonics News 9(11), $40-41$ (1998) 